\title{
ENSAIOS DE TRAÇÃO COMPARATIVOS ENTRE COMPÓSITOS REFORÇADOS COM CURAUÁ E COMPÓSITOS REFORÇADOS COM FIBRAS DE VIDRO*
}

\section{Resumo}

Felipe Perissé Duarte Lopes ${ }^{1}$ Janaína da Silva Vieira ${ }^{2}$

Frederico Muylaert Margem ${ }^{3}$ Sergio Neves Monteiro ${ }^{4}$

Carlos Maurício Pontes Vieira ${ }^{5}$ Luís Carlos da Silva ${ }^{6}$

Atualmente, uma das principais preocupações da humanidade está relacionada à questões ambientais, que motivam pesquisas para o desenvolvimento de materiais com recursos renováveis e amigáveis com o meio ambiente. No entanto, este estudo pretende comparar, à resistência dos compósitos reforçados com fibras naturais, portanto, favoráveis ao meio ambiente e compósitos reforçados com fibras de vidro, que por sua vez têm impactos ambientais desde a fabricação até à disposição. Treze compósitos foram confeccionados para essa pesquisa, contendo $0 \%$ de fibra, $30 \%$ de fibras de curauá e $30 \%$ de fibras de vidro em matriz epóxi e testados em uma máquina de tração. Os resultados mostraram valores mais altos para as amostras reforçadas com fibras de vidro, no entanto, para relacionar a tensão do compósito com à sua densidade de fibra, verificouse que os compósitos reforçados com fibras naturais apresentaram resultados melhores do que os produzidos com fibras de vidro. Concluindo, pelo ponto de vista da propriedade mecânica, as fibras de vidro podem ser substituídas pelas fibras de curauá.

Palavras-chave: Ensaios de tração; Fibras de curauá; Fibras de vidro; compósito; Epóxi.

\section{COMPARATIVE TENSILE TEST ANALYSIS BETWEEN EPOXY COMPOSITES REINFORCED WITH CURAUA FIBER AND FIBERGLASS}

\begin{abstract}
Currently, one of the major humanity concerns is related to environmental issues, which has motivated researches and materials developments from renewable and environmental friendly resources. Nevertheless, this study aims to compare, the composites resistance developed with natural fibers, therefore environmental friendly and composites developed with fiberglass, which in turn have environmental impacts from manufacturing to disposal. Thirteen specimens were fabricated to test, what containing $0 \%$ fiber, $30 \%$ curaua fiber and $30 \%$ fiberglass in epoxy matrix and tested in tensile machine. The results show higher values for the samples containing fiberglass, however, to relate the composite tension with its fiber density, it was found that the composites with natural fibers even showed better results than those produced composites with fiberglass. Concluding, from the point of view of mechanical property, fiberglass can be replaced by curaua fiber.
\end{abstract}

Keywords: Tensile test, curaua fiber, fiberglass, composite, epoxy.

1 Engenheiro Civil, M.sc., Mestre em Ciência dos Materiais, Seção de Engenharia Mecânica e de Materiais, Instituto Militar de Engenharia, Rio de Janeiro, RJ Brasil.

2 Engenheiro Civil, M.sc., Mestre em Ciência dos Materiais, Seção de Engenharia Mecânica e de Materiais, Instituto Militar de Engenharia, Rio de Janeiro, RJ Brasil.

3 Engenheiro Civil, M.sc., Mestre em Ciência dos Materiais, Seção de Engenharia Mecânica e de Materiais, Instituto Militar de Engenharia, Rio de Janeiro, RJ Brasil.

4 Engenheiro Metalurgista, Ph.D, Professor Titular, Seção de Engenharia Mecânica e de Materiais, Instituto Militar de Engenharia, Rio de Janeiro, RJ, Brasil.

5 Engenheiro Metalurgista, Ph.D, Professor Titular, Seção de Engenharia Mecânica e de Materiais, Instituto Militar de Engenharia, Rio de Janeiro, RJ, Brasil.

6 Engenheiro Químico, D.Sc., Doutor em Ciência dos Materiais, Seção de Engenharia Mecânica e de Materiais, Instituto Militar de Engenharia, Rio de Janeiro, RJ, BrasilFormação/graduação, titulação, cargo/função, setor/departamento, Instituição de trabalho e/ou estudo, cidade, estado e país. 


\section{INTRODUÇÃO}

Os materiais compósitos modernos, particularmente os reforçados com fibras sintéticas, como a fibra de vidro e a fibra de carbono por exemplo, têm sido utilizados desde o século passado para atender às exigências em muitos campos tecnológicos, desde o desenvolvimento de eletrodomésticos até peças aeroespaciais [1]. No entanto, a energia necessária para produzir essas fibras gera uma grande emissão de gases de $\mathrm{CO}_{2}$, que é o fator responsável pelo aquecimento global. Além disso, a fibra de vidro não é reciclável e não pode ser incinerada, e os seus resíduos não podem ser descartados em aterros sanitários, contribuindo assim para à poluição ambiental [2]. Atualmente, as preocupações ecológicas tornaram-se relevantes para à sociedade visando uma melhora de qualidade da vida das populações, o que exigiu das empresas uma nova posição em sua interação com o meio ambiente [3], buscando formas alternativas de blindagem para substituir, sempre que possível, as fibras sintéticas pelas fibras naturais, uma vez que estas têm custos mais baixos, são renováveis e biodegradáveis e o seu sistema de produção não exige o uso de muitos equipamentos e são eficientes em termos energéticos [4]. A TABELA 1 resume as principais vantagens do uso de fibras naturais em comparação com as fibras de vidro.

Tabela1. Comparações entre a fibra natural e a fibra de vidro

\begin{tabular}{|l|c|c|}
\hline & FIBRAS NATURAIS & FIBRAS DE VIDRO \\
\hline Densidade & Baixa & $\begin{array}{c}\text { O dobro do valor da fibra } \\
\text { natural }\end{array}$ \\
\hline Custo & Baixo & $\begin{array}{c}\text { Alto, se comparado com a fibra } \\
\text { natural }\end{array}$ \\
\hline Renovável & Sim & Não \\
\hline Reciclável & Sim & Não \\
\hline Energia consumida & Baixa & Alta \\
\hline $\mathrm{CO}_{2}$ (Neutro) & Sim & Sim \\
\hline $\begin{array}{l}\text { Abrasivo para as } \\
\text { máquinas }\end{array}$ & Não & Sim \\
\hline $\begin{array}{l}\text { Risco de saúde quando } \\
\text { inalado }\end{array}$ & Não & Não biodegradável \\
\hline Degradação & Biodegradável \\
\hline
\end{tabular}

Segundo Ledo [5-6] O curauá se destaca, entre as fibras naturais com aplicações potenciais em compósitos, devido à excelente qualidade de suas fibras além de ser usado na indústria automobilística, devido à sua boa resistência, suavidade e baixo peso. O cientista japonês Junpei Kanazashi em sua pesquisa, descobriu que as fibras de curauá possuem alta resistência mecânica comparadas com outras fibras catalogadas no mercado global [7]. O objetivo principal deste estudo é comparar o comportamento mecânico, especificamente a resistência à tração de compósitos poliméricos reforçados com fibras naturais de curauá com compósitos poliméricos reforçados com fibras de vidro. 


\section{MATERIAIS E MÉTODOS}

\subsection{Fibras de Curauá}

As fibras de curauá foram obtidas a partir de uma espécie de planta cultivada na Região Norte do Brasil com o mesmo nome, uma bromélia (Ananas Erectifolius), da família do abacaxi e foi comprada de uma empresa especializada em fibras lignocelulósicas, chamada "Amazon Paper [4, 8- 11]. O aspecto típico dessa planta e das fibras de curaua são mostrados na FIGURA 1 abaixo.

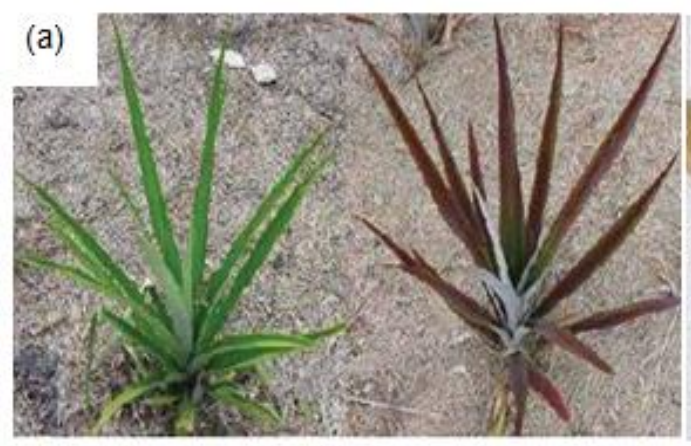

\section{(b)}

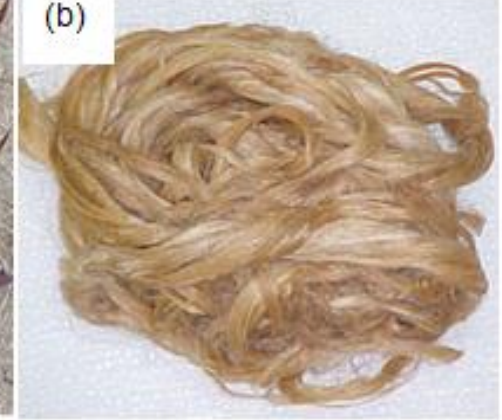

FIGURA 1. (a) Planta Curauá e (b) Fibras de Curauá processadas e prontas para ser usada

As fibras foram lavadas e secas ao ar livre sem nenhum tratamento superficial. 100 fibras foram selecionadas e os seu comprimentos e diâmetros foram medidos através de uma régua e um projetor de perfil, e com os resultados obtidos foram calculados o comprimento médio (L) e o diâmetro médio (d). A FIGURA 2 demonstra o histograma onde foi possível obter $L=442 \mathrm{~mm}$ e $\mathrm{d}=0,17 \mathrm{~mm}$, aproximadamente [4]. As fibras foram pesadas individualmente e através das suas medidas geométricas, foi possível calcular a densidade média da fibra de curauá, que é de aproximadamente $0,92 \mathrm{~g} / \mathrm{cm}^{3}$ [7].
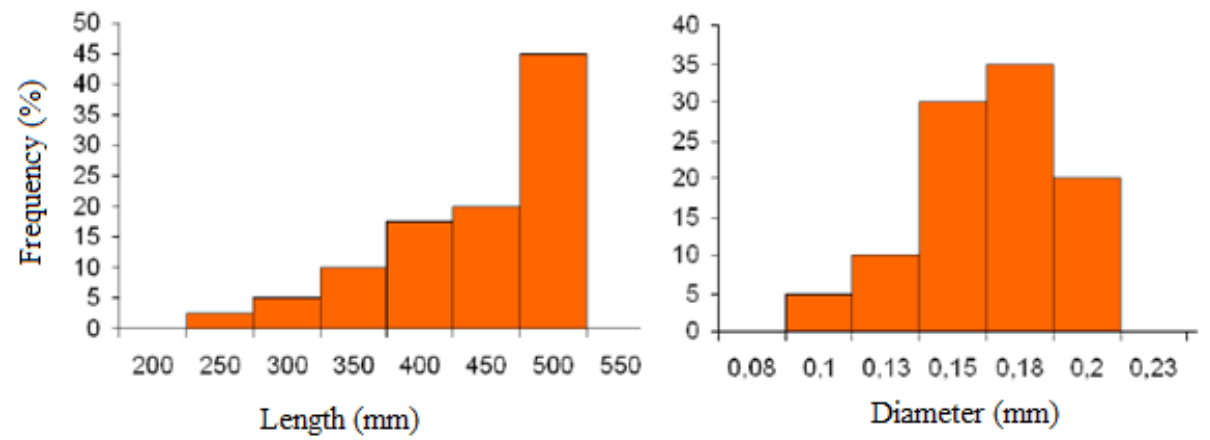

FIGURA 2. Histograma demonstrando a distribuição de comprimento e diâmetro das fibras de curauá

\subsection{Fibras de vidro}

As fibras de vidro foram compradas da "Glass Company". Uma empresa que trabalha com reparos em veículos e pranchas de surf usando como matéria-prima a fibra de vidro. De acordo com o fabricante, a fibra de vidro é do tipo $\mathrm{E}$ com uma densidade de aproximadamente $2,6 \mathrm{~g} / \mathrm{cm}^{3}$ e um diâmetro médio na faixa de 8 a $14 \mathrm{~mm}$. 


\subsection{Experimentos}

Para realizar este trabalho, foram feitos 13 ensaios de tração para cada concentração de fibra no compósito: $0 \%$ de fibra, 30\% fibra de curauá (FC) e 30\% de fibra de vidro (FV) em matriz epóxi. Os compósitos com $0 \%$ de fibra foram feitos em matriz de silicone. No caso das amostras com $30 \%$ de fibra, as fibras foram cortadas no tamanho da amostra, alinhadas, inseridas na matriz metálica e depois banhadas em resina epóxi. Todos os compósitos foram processados à temperatura ambiente e depois foram submetidos à uma pressão de 2,5 toneladas por 24 horas durante o período de cura. Após a cura, as amostras foram desmoldadas com o auxílio de uma pinça, os corpos de prova foram medidos, numerados e submetidos a um ensaio de tração usando a máquina INSTRON Modelo 5582 com uma velocidade de $2 \mathrm{~mm} / \mathrm{min}$ à temperatura ambiente. A FIGURA 3 demonstra os passos descritos acima.

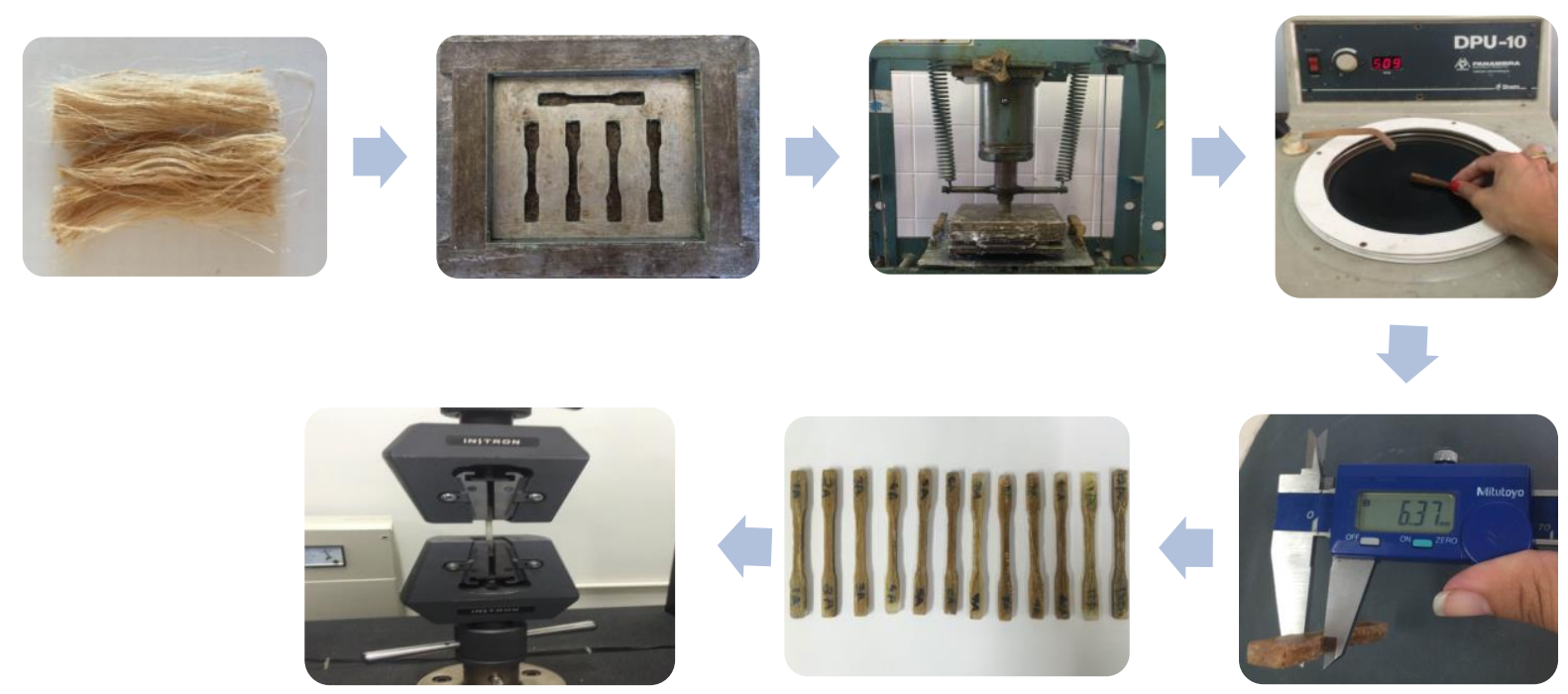

FIGURA 3. Procedimento de fabricação dos compósitos reforçados com $30 \%$ de fibra de curauá para o ensaio de tração.

\section{RESULTADOS E DISCUSSÃO}

A FIGURA 4 ilustra a macroestrutura da região de fratura das diferentes amostras. Nesta figura, a fratura correspondente as amostras de epóxi puro tende a ser transversal à direção de tração. Este fato indica que o mecanismo de fratura para a matriz epóxi pura é associado à propagação de trincas transversais. No entanto, com o reforço da fibra de curauá a fratura tende a mudar de direção porque a fratura segue a fibra e esse comportamento está associado a uma baixa adesão entre as fibras de curauá e a matriz de epóxi. A amostra contendo fibras de vidro não fraturou. 
$\bar{\omega}$
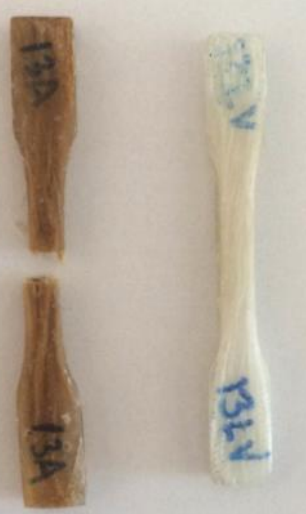

FIGURA 4. Fratura das amostras contendo $0 \%$ de fibras, $30 \%$ de fibras de curauá e $30 \%$ de fibras de vidro reforçando uma matriz de epóxi

A Figura 5 mostra um gráfico dos resultados de ensaios de tração para as amostras contendo $0 \%$ de fibra, $30 \%$ de $\mathrm{FC}$ e $30 \%$ de FV, respectivamente.

\section{Resistência a tração de três diferentes amostras}

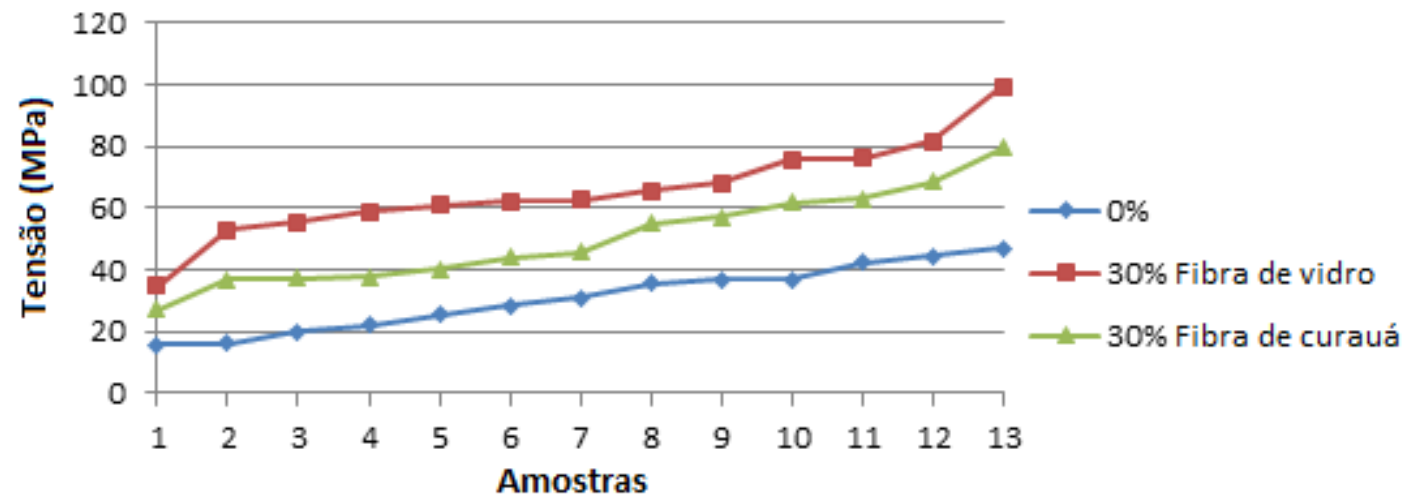

FIGURA 5. Gráfico comparativo de resistência a tração de amostras contendo $0 \%$, $30 \% \mathrm{FC}$ e $30 \% \mathrm{FV}$

É possível observar um aumento significativo de resistência à tração quando a matriz de epóxi é reforçada com as fibras [8]. A resistência a tração de amostras com $30 \%$ de FV foi maior do que a resistência a tração de amostras de FC, esse fenômeno é esperado, uma vez que as fibras naturais por serem hidrofílicas têm pouca compatibilidade com a matriz que é hidrofóbica, resultando em baixa adesão na interface entre a fibra e a matriz, influenciando diretamente as propriedades mecânicas do compósito [9]. Além disso, as propriedades mecânicas e as propriedades físicas das fibras naturais variam consideravelmente pois dependem da composição química e estrutural e das condições de crescimento das plantas, dessa forma as fibras naturais possuem grandes dispersões em suas propriedades comparando-as com as fibras de vidro. Essa alta dispersão contribui para resultados inferiores de resistência em comparação com materiais homogêneos como a fibra de vidro [10]. Embora materiais biodegradáveis (materiais verdes) apresentem propriedades inferiores em relação as propriedades dos materiais sintéticos, seu uso em escala industrial não é afetado [11]. 
Devido ao fato dos materiais estudados terem essa alta dispersão, foi necessário avaliar a resistência de acordo com a densidade de cada material, dessa forma podese avaliar a tensão de acordo com a densidade de cada material, conforme mostrado no gráfico da FIGURA 6.

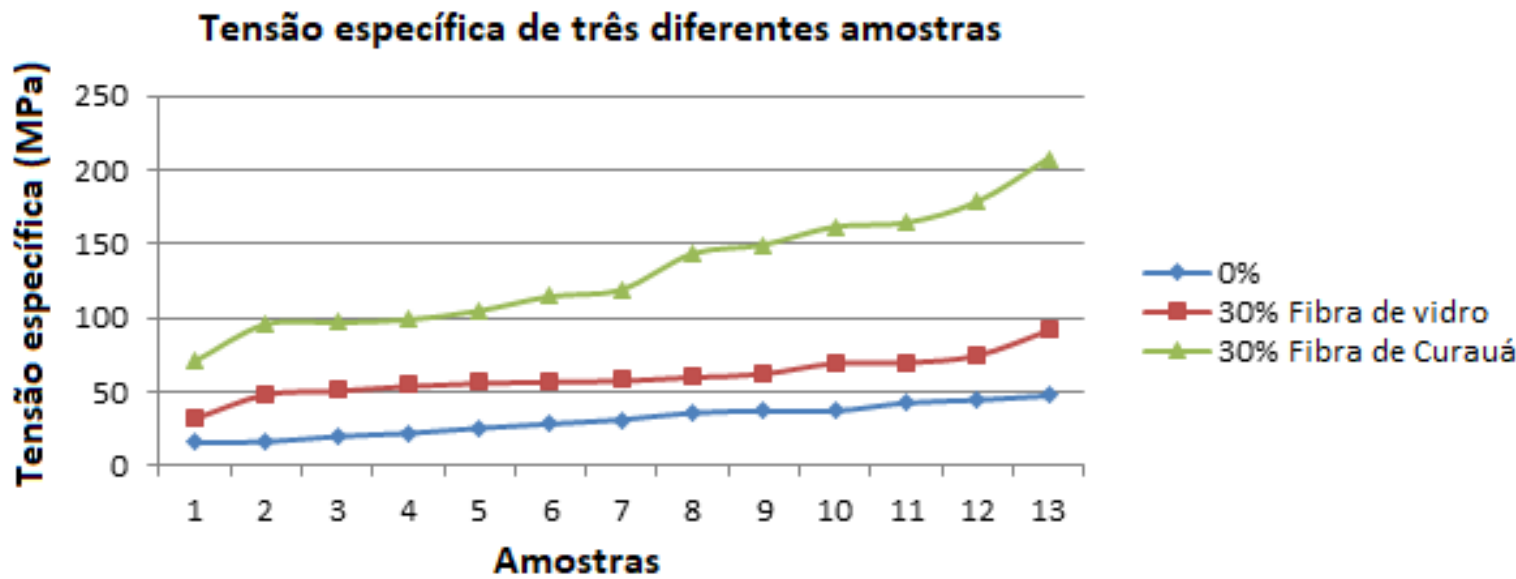

FIGURA 6. Gráfico comparativo de tensão específica de amostras contendo 0\%, 30\% FC e $30 \%$ FV

Ao dividir a resistência à tração do material pela sua densidade obtem-se à tensão específica, os compósitos de FC possuem valores mais altos dessa razão do que os compósitos de FV. As propriedades mecânicas das fibras naturais são bem inferiores quando comparadas com as fibras mais amplamente utilizadas como a fibra de vidro. No entanto, devido à sua baixa densidade, as propriedades específicas (razão propriedade/densidade), resistência e rigidez das fibras naturais são comparáveis aos valores da fibra de vidro². É importante reafirmar que as dispersões de alguns dos resultados são significativas. Isso pode ser explicado pela dificuldade na preparação uniforme das amostras. A TABELA. 2 mostra a média das amostras com $0 \%$ de fibra, $30 \%$ de $\mathrm{FC}$ e $30 \%$ de FV.

\begin{tabular}{cccccc}
\hline & $\begin{array}{c}0 \% \\
(\mathrm{MPa})\end{array}$ & $\begin{array}{c}30 \% \\
\mathrm{CF} \\
(\mathrm{MPa})\end{array}$ & $\begin{array}{c}30 \% \\
\mathrm{FV} \\
(\mathrm{MPa})\end{array}$ & $\begin{array}{c}30 \% \text { CF Tensão } \\
\text { específica } \\
(\mathrm{MPa})\end{array}$ & $\begin{array}{c}30 \% \text { FV Tensão } \\
\text { específica } \\
(\mathrm{MPa})\end{array}$ \\
\hline Média & 30.96 & 60.57 & 130.84 & 65.84 & 50.32 \\
\hline
\end{tabular}

Ao adicionar fibras de curauá em resina epóxi, houve um aumento de $100 \%$ da resistência. Para a fibra de vidro, o aumento foi ainda mais significativo, ou seja, a resistência aumentou $100 \mathrm{MPa}$ em comparação com a amostra sem fibra epóxi. Comparando os resultados de FC e FV, percebe-se que a resistência mais do que duplicou, no entanto, para fazer uma análise mais crítica, fica evidente que a resistência de FC é maior do que o FV quando leva-se em consideração a densidade dos dois materiais. 


\section{CONCLUSÃO}

A quantidade crescente de fibras tem influência direta sobre o comportamento mecânico de compósitos reforçados com fibras naturais. A amostra de epóxi puro tem menor resistência que o compósito contendo $30 \%$ de fibra. Os resultados mostram que o compósito com fibra de vidro é mais interessante. No entanto, quando analisase os resultados com a razão tensão/densidade é possível concluir que o compósito de fibras de curauá é mais resistente que o compósito de fibras de vidro.

\section{REFERÊNCIAS}

1 Chawla KK. Composite Materials Science and Engineering. 2nd edition. New York: Springer-Verlag; 1998.

2 Wambua P, Ivens I, Verpoest I. "Natural fibers: can they replace glass and fibre reinforced plastics?". Composites Science and Technology. 2003; 63: 12591264.

3 Donaire D. Considerações sobre a influência da variável ambiental na empresa. Revista de Administração de Empresas. 1994; 34(2): 68-77.

4 Monteiro SN, Lopes FPD, Ferreira AS, Nascimento DCO. Natural fiber polymer matrix composites: Cheaper, tougher, and environmentally friendly. JOM. 2009; 61(1):17-22.

5 Ledo IAM. O cultivo no Lago Grande da França. 1º edição. Belém: Banco de Crédito da Amazônia S/A; 1964.

6 S.N. Monteiro, K.G. Satyanarayana, F.P.D. Lopes, "High strength natural fibers for improved polymer matrix composites", Mater. Sci. Forum, 638-642 (2010) 961-966.

7 Takahashi R, Pereira LCO, Oliveira DRC, Fujiyama RT. Fabricação de mantas de fibra de sisal e de curauá. COBENGE. 2011 [acesso em 24 Fev. 2014]. Disponível 